\title{
A Web Interface for Matita
}

\author{
Andrea Asperti and Wilmer Ricciotti \\ Department of Computer Science, University of Bologna \\ \{asperti,ricciott\}@cs.unibo.it
}

This article describes a prototype implementation of a web interface for the Matita proof assistant [2]. The motivations behind our work are similar to those of several recent, related efforts [7/9|18] (see also [6]). In particular:

1. creation of a web collaborative working environment for interactive theorem proving, aimed at fostering knowledge-intensive cooperation, content creation and management;

2. exploitation of the markup in order to enrich the document with several kinds of annotations or active elements; annotations may have both a presentational/hypertextual nature, aimed to improve the quality of the proof script as a human readable document, or a more semantic nature, aimed to help the system in its processing (or re-processing) of the script;

3. platform independence with respect to operating systems, and wider accessibility also for users using devices with limited resources;

4. overcoming the installation issues typical of interactive provers, also in view of attracting a wider audience, especially in the mathematical community.

Point 2. above is maybe the most distinctive feature of our approach, and in particular the main novelty with respect to [7].

In fact, delivering a proof assistant as a web application enables us to exploit the presentational capabilities of a web browser with little effort. Purely presentational markup does not require any special treatment on the part of the prover and is natively supported by the web browser. However, having an easy access to HTML-like markup allows much more flexibility. Not only can we decorate comments by means of textual formatting or pictures; executable parts of scripts reference concepts defined elsewhere, either in the same script or in the library, using possibly overloaded identifiers or notations: it is natural to enrich those identifiers with hyperlinks to the associated notions. This association is actually computed by the system every time the script is parsed, hence it is the system's job to enrich the script accordingly. Since the previous computation can be expensive, it is natural to have the system use such hyperlinks to speed up the execution of the script. Moreover, when the source text is particularly ambiguous, hyperlinks provide essential semantic information to avoid asking the user for explicit disambiguation every time the script is executed.

Hyperlinks are an example of a textual annotation having both a presentational and a semantic value. The text enriched with hyperlinks not only provides 
a more dynamic and flexible format to access the repository, but it is also a more explicit and hence more robust representation of the information.

A further use of markup is to attach to the script information that is valuable to the system, but is not thought to be normally read by the user. This is technically a kind of presentational markup, used to hide parts of the script rather than for decorating text.

Our current implementation supports three categories of markup:

- standard HTML markup, used to add formatting to text; formatted text is currently assumed to occur in Matita comments;

- hyperlinks to Matita definitions, typically produced by the system and reused on a new parsing of the script to avoid a second disambiguation of the input (at the time of the submission, traversing hyperlinks is not yet supported, but implementing it does not look problematic);

- markup wrapping traces of execution of automation steps in the script, produced by the system on a first execution and granting a notable speed-up on future executions; the trace is normally transparent to the user, but visible on demand.

\section{Structure of the system}

Matita core The server runs a minimally reworked version of the Matita engine, equivalent to its stand-alone counterpart, but for the following features:

- the status of Matita includes the user id of its owner, as needed by an inherently multi-user web application: this allows the system to run at the same time several user-specific versions of the library;

- the lexical analyzer and the parser take into account the script markup;

- the disambiguation engine and the automation tactic produce and return information suitable for enriching the script.

For what concerns the lexical analyzer, producing specific tokens for the markup would require major modifications to the parser, which in Matita is a complex component extensible at runtime with user provided notations. In an effort to keep the parser as untouched as possible, the token stream returned by our lexical analyzer ignores the markup; however, hyperlinks that can be used for immediate disambiguation are stored in an additional table that is later accessible to the parser, which is then able to build a disambiguated abstract syntax tree (AST) for it. In order for this technique to work, we assume that disambiguation markup is only located around "leaves" of the AST (and in particular, identifiers or symbols); at the moment, this assumption does not seem to be restrictive.

Markup for automation traces, which is used only to hide additional arguments to the automation tactic, is completely handled by the user interface and can thus be safely ignored by the lexical analyzer and the parser. 
Matita web daemon The Matita web daemon is a specialized HTTP server, developed using the Netplex module of the Ocamlnet library 1], providing remote access to the Matita system. It exports several services:

- storage of user accounts and authentication;

- storage of user libraries;

- synchronization of user libraries with the shared library via svn;

- remote execution of scripts.

Such services are invoked through a CGI interface and return XML documents encoding their output.

Remote execution of scripts allows a user authoring a script on a web browser to send it to the server for processing. The typical interactions with a script are allowed, in the style of Proof-General [4] and similarly to [7]: executing one step (tactic or directive) or the whole script, as well as undoing one step or the whole script (execution of a script until a given point is reached is performed by the client by multiple calls to single-step execution).

Parsing of the script is performed on the server, as client-side parsing of the extensible syntax used by Matita is essentially unfeasible. To execute (part of) a script, the server needs thus to receive all of the remaining text to be parsed, because the end of the next statement is not predictable without a full parsing. The Matita daemon will answer such a request by returning to the client

- the length of the portion of the original script that has been successfully executed;

- a (possibly empty) list of parsed statements, which have been enriched with mechanically generated markup including disambiguation hints and automation traces (the length of this updated text does not match the previous value in general);

- an HTML representation of the proof state of the system after the execution of the last statement (if the execution stopped in the middle of a proof);

- a representation of the error that prevented a further execution of the script (if the execution stopped because of an error).

Collaborative formalization The daemon provides a preliminary support for collaborative formalization, currently coming in the form of a centralized library maintained by means of svn. Other authors (see [1]) have advocated the use of distributed versioning systems (e.g. Git). Our choice is mainly related to the reuse of the original Matita repository and to the fact that svn already supports the kind of distributed activity we have in mind. The effective usability and scalability of this approach will be tested in the future.

The client The Matita web client was initially written in plain Javascript and is currently being ported to the jQuery 2 framework. The client implements a

${ }^{1}$ http://projects.camlcity.org/projects/ocamlnet.html

2 http://jquery.com 
user interface that is essentially similar to the one of ProofGeneral 4, CtCoq and CoqIDE [5], or stand-alone Matita [3], but in the form of a web page. This includes displaying the script (disabling editing for the already executed part), buttons for script navigation, boxes for proof state (including multiple open goals) and disambiguation, instant conversion of $\mathrm{T}_{\mathrm{E}} \mathrm{X}$-like escapes to Unicode symbols, and essential interface for accessing the remote file system.

The implementation issues are similar to those described in [10]. The web interface does not need to understand much of Matita: information like being in an unfinished proof or in disambiguation mode can be easily inferred from the data structures returned from the server. On the other hand, some code is necessary to convert Matita markup to HTML markup and vice-versa.

Availability The Matita web interface is accessible from the website http://pandemia.helm.cs.unibo.it/login.html. Accounts for accessing the interface are provided by the authors on request.

\section{References}

1. Jesse Alama, Kasper Brink, Lionel Mamane, and Josef Urban. Large formal wikis: Issues and solutions. In Proceedings of Intelligent Computer Mathematics (CICM 2011), Bertinoro, Italy, volume 6824 of Lecture Notes in Computer Science, pages 133-148. Springer, 2011.

2. Andrea Asperti, Wilmer Ricciotti, Claudio Sacerdoti Coen, and Enrico Tassi. The Matita interactive theorem prover. In Proceedings of the 23rd International Conference on Automated Deduction (CADE-2011), Wroclaw, Poland, volume 6803 of LNCS, 2011.

3. Andrea Asperti, Claudio Sacerdoti Coen, Enrico Tassi, and Stefano Zacchiroli. User interaction with the Matita proof assistant. Journal of Automated Reasoning, 39(2):109-139, 2007.

4. David Aspinall. Proof General: A generic tool for proof development. In Tools and Algorithms for the Construction and Analysis of Systems, TACAS 2000, volume 1785 of Lecture Notes in Computer Science. Springer-Verlag, January 2000.

5. Yves Bertot and Laurent Théry. A generic approach to building user interfaces for theorem provers. Journal of Symbolic Computation, 25:161-194, 1998.

6. Herman Geuvers. Proof Assistants: history, ideas and future. Sadhana, 34(1):3-25, 2009 .

7. Cezary Kaliszyk. Web interfaces for proof assistants. Electr. Notes Theor. Comput. Sci., 174(2):49-61, 2007.

8. C. Tankink, H. Geuvers, J. McKinna, and F. Wiedijk. Proviola: A tool for proof re-animation. In Proceedings of AISC 2010, Heidelberg, volume 6167 of Lecture Notes in Computer Science, pages 440-454. Springer, 2010.

9. Josef Urban, Jesse Alama, Piotr Rudnicki, and Herman Geuvers. A wiki for mizar: Motivation, considerations, and initial prototype. CoRR, abs/1005.4552, 2010.

10. Makarius Wenzel. Isabelle as document-oriented proof assistant. In Intelligent Computer Mathematics - 18th Symposium, Calculemus 2011, and 10th International Conference, MKM 2011, Bertinoro, Italy, July 18-23, 2011. Proceedings, volume 6824 of $L N C S$, pages 244-259, 2011. 